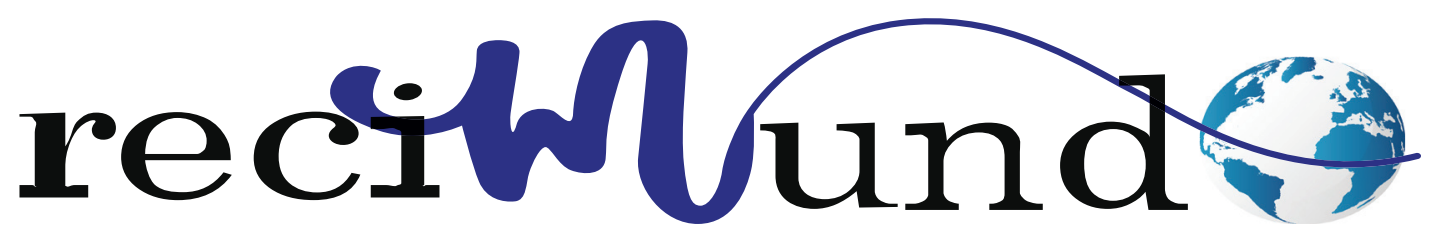

Revista Científica Mundo de la Investigación y el Conocimiento

DOI: 10.26820/recimundo/5.(3).sep.2021.424-432

URL: https://recimundo.com/index.php/es/article/view/1324

EDITORIAL: Saberes del Conocimiento

REVISTA: RECIMUNDO

ISSN: 2588-073X

TIPO DE INVESTIGACIÓN: Artículo de revisión

CóDIGO UNESCO: 32 Ciencias Médicas

PAGINAS: $424-432$

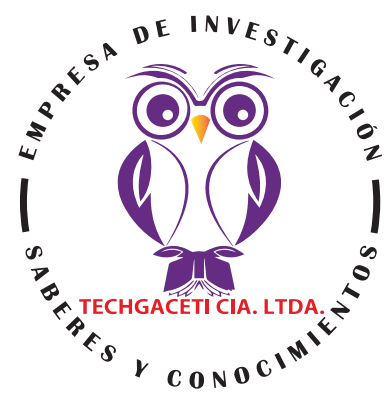

\title{
Causas y consecuencias de los trastornos de la tiroides
}

Causes and consequences of thyroid disorders

Causas e consequências dos distúrbios da tireoide

Fabián Marcelo Zambrano Palacios'; María Lissette Soledispa Zambrano²; Genesis Monserrate Demera Reyna3; Jonathan David Alvarado Cedeño ${ }^{4}$

RECIBIDO: 10/08/2021 ACEPTADO: 15/08/2021 PUBLICADO: 30/09/2021

1. Médico General; Médico General en Funciones Hospitalarias; Hospital Dr. Gustavo Domínguez; Santo Domingo de los Tsáchilas, Ecuador; fabianzambrano95@hotmail.com; https://orcid.org/0000-0002-1804-6408

2. Médico General; Médico General en Funciones Hospitalarias; Hospital Dr. Gustavo Domínguez; Santo Domingo de los Tsáchilas, Ecuador; lissolezambrano90@hotmail.com; https://orcid.org/0000-0001-7469-5712

3. Médico General; Médico General en Funciones Hospitalarias; Hospital Dr. Gustavo Domínguez; Santo Domingo de los Tsáchilas, Ecuador; genesis2244@hotmail.com; https://orcid.org/0000-0001-7785-2066

4. Médico General; Atención primaria en Salud; Medinorte; Quito, Ecuador; jondavid_17@hotmail.com; https://orcid.org/00000003-1978-9892

CORRESPONDENCIA

Fabián Marcelo Zambrano Palacio

fabianzambrano95@hotmail.com

Quito, Ecuador

(C) RECIMUNDO; Editorial Saberes del Conocimiento, 2021 


\section{RESUMEN}

Millones de personas, pueden padecer algún trastorno de la tiroides, pero su diagnostico en muchos casos puede ser complejo, ya que los síntomas están mas asociados a los estilos de vida de las personas, las causas pueden ser diversas, como es el caso de la falta o exceso de yodo que pueden producir alteraciones de nódulos y enfermedades autoinmunes, la enfermedad de Graves es una de las causantes del hipertiroidismo y así otras enfermedades que pueden generar otras alteraciones de la tiroides. La metodología utilizada para el presente trabajo de investigación, se enfoca hacia una metodología enfocada hacia la necesidad de indagar en forma precisa y coherente una situación. Enmarcada dentro de una revisión bibliográfica de tipo documental, ya que nos vamos a ocupar de temas planteados a nivel teórico como es causas y consecuencias de los trastornos de la tiroides. La técnica para la recolección de datos está constituida por materiales impresos, audiovisuales y electrónicos, estos últimos como Google Académico, PubMed, entre otros. La información aquí obtenida será revisada para su posterior análisis. Las hormonas tiroideas son muy importantes, ya que regulan muchas funciones y procesos metabólicos, para que las hormonas tiroideas funciones normalmente, es necesario que la glándula tiroidea se desarrolle normalmente, y para que esto suceda es necesario que haya un soporte nutricional de yodo adecuado, la propia autorregulación tiroidea, el desarrollo normal de los procesos controlados y regulados por el hipotálamo-hipofisario, entre otros. Y esto es importante, ya que el eje hipotálamo-hipófisis-tiroides, regulan las hormonas tiroideas, que permiten mantener un aporte de hormonas adecuado al sistema nervioso, el exceso o deficiencia de estas hormonas pueden producir alteraciones criticas en la vida intrauterina, los primeros años de vida, adolescencia y la etapa adulta.

Palabras clave: Hormonas, Tiroides, Cáncer, Nódulos, Hipertiroidismo.

\section{ABSTRACT}

Millions of people can suffer from a thyroid disorder, but its diagnosis in many cases can be complex, since the symptoms are more associated with people's lifestyles, the causes can be diverse, such as lack or excess of iodine that can cause alterations of nodules and autoimmune diseases, Graves' disease is one of the causes of hyperthyroidism and thus other diseases that can generate other alterations of the thyroid. The methodology used for this research work is focused on a methodology focused on the need to investigate a situation precisely and coherently. Framed within a bibliographic review of a documentary type, since we are going to deal with issues raised at a theoretical level such as causes and consequences of thyroid disorders. The technique for data collection is made up of printed, audiovisual and electronic materials, the latter such as Google Scholar, PubMed, among others. The information obtained here will be reviewed for further analysis. Thyroid hormones are very important, since they regulate many functions and metabolic processes, for thyroid hormones to function normally, it is necessary for the thyroid gland to develop normally, and for this to happen it is necessary that there is adequate nutritional support of iodine, the thyroid self-regulation itself, the normal development of the processes controlled and regulated by the hypothalamus-pituitary, among others. And this is important, since the hypothalamic-pituitary-thyroid axis regulates thyroid hormones, which allow maintaining an adequate supply of hormones to the nervous system, the excess or deficiency of these hormones can produce critical alterations in intrauterine life, the former years of life, adolescence and adulthood.

Keywords: Hormones, Thyroid, Cancer, Nodules, Hyperthyroidism.

\section{RESUMO}

Milhões de pessoas podem sofrer de algum distúrbio da tireoide, mas seu diagnóstico em muitos casos pode ser complexo, já que os sintomas estão mais associados ao estilo de vida das pessoas, as causas podem ser diversas, como falta ou excesso de iodo que podem causar alterações dos nódulos e doenças autoimunes, a doença de Graves é uma das causas do hipertireoidismo e, portanto, de outras doenças que podem gerar outras alterações da tireoide. A metodologia utilizada para este trabalho de investigação centra-se numa metodologia centrada na necessidade de investigar uma situação de forma precisa e coerente. Enquadrado dentro de uma revisão bibliográfica de tipo documental, visto que trataremos de questões levantadas a nível teórico como causas e consequências dos distúrbios da tireoide. A técnica de coleta de dados é composta por materiais impressos, audiovisuais e eletrônicos, estes últimos como Google Scholar, PubMed, entre outros. As informações aqui obtidas serão revisadas para análises posteriores. Os hormônios tireoidianos são muito importantes, pois regulam várias funções e processos metabólicos, para que os hormônios tireoidianos funcionem normalmente é necessário que a glândula tireoide se desenvolva normalmente, e para que isso aconteça é necessário que haja suporte nutricional adequado de iodo, a própria autorregulação da tireoide, o desenvolvimento normal dos processos controlados e regulados pelo hipotálamo-hipófise, entre outros. E isso é importante, uma vez que o eixo hipotálamo-hipófise-tireoide regula os hormônios tireoidianos, que permitem manter um suprimento adequado de hormônios ao sistema nervoso, o excesso ou a deficiência desses hormônios podem produzir alterações críticas na vida intrauterina, os primeiros anos de vida. , adolescência e idade adulta.

Palavras-chave: Hormônios, tireóide, câncer, nódulos, hipertireoidismo. 
ÑO, J. D.

\section{Introducción}

La glándula tiroides, situada justo por debajo de la laringe y a ambos lados y por delante de la tráquea, es una de las glándulas endocrinas más grandes, con un peso que oscila entre 15 y 20 gramos en los adultos sanos, está formada por dos mitades (lóbulos) conectadas en su parte central (istmo). Si aumenta de tamaño, se palpa con facilidad como un bulto prominente (bocio) que aparece debajo o a los lados de la nuez de Adán (Yeza \& Noemí, 2020, pág. 6).

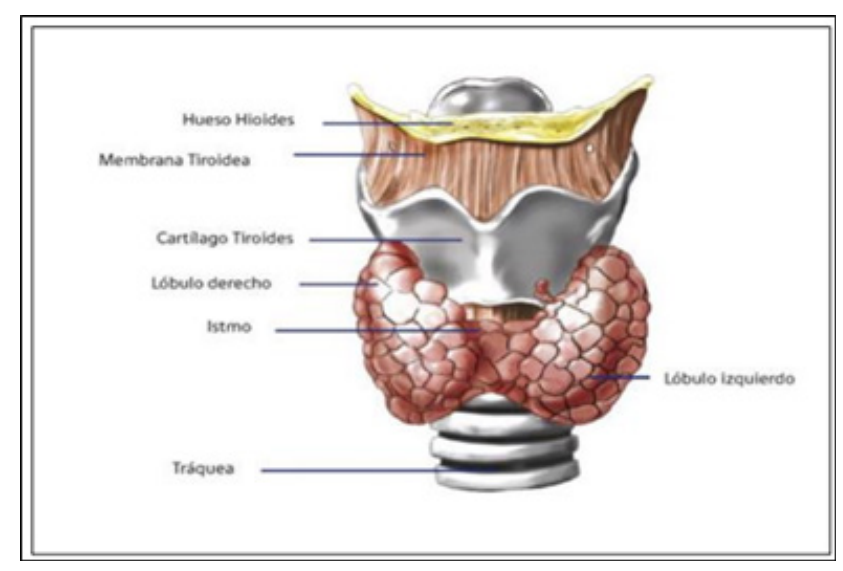

Imagen 1. Localización y relaciones anatómicas de la glándula tiroides.

Fuente: (Yeza \& Noemí, 2020)

La disfunción tiroidea subclínica (DTS) es un trastorno funcional de la glándula tiroides de alta prevalencia, asintomática y solo detectable mediante exámenes hormonales de laboratorio, la cual puede corresponder a una enfermedad tiroidea en su etapa inicial, especialmente en individuos de mediana y avanzada edad, manifestándose por concentraciones alteradas de hormona estimulante de tiroides (TSH) y concentraciones séricas normales de triyodotironina (T3) y tiroxina (T4) libres (Palacio, y otros, 2017). Alrededor de 700 millones de personas en el mundo padecen algún trastorno de tiroides. Sin embargo, en algunos países el 50\% de ellas no lo saben, ya que los síntomas pueden ser confundidos con su estilo de vida. Según estadísticas de la Organización Mundial de la Salud (OMS), afecta al $10 \%$ de la población mundial y la gran mayoría desconoce los síntomas de esta condición. Así lo reveló la más reciente encuesta de la Federación Internacional de la Tiroides (TFI), por sus siglas en inglés) y Merck, empresa líder en ciencia y tecnología para el cuidado de la salud, con el objetivo de generar conciencia en el marco de la semana de esta importante glándula del organismo (David \& Cuvi, 2019, pág. 1).

Las enfermedades tiroideas son comunes en la población mundial, aunque su prevalencia varía de acuerdo con el área geográfica. Las alteraciones tiroideas como los nódulos son más prevalentes en áreas con déficit de yodo, mientras que las enfermedades autoinmunes son más frecuentes en áreas repletas de yodo. No obstante, existen muchos más factores externos que inciden en el surgimiento de estas alteraciones, como es la radiación ionizante. Estas disfunciones son fáciles de identificar y tratar, sin embargo, de no ser diagnosticadas y tratadas a tiempo pueden generar consecuencias profundas en las personas y en una población en particular (Rosero López, 2020, pág. 4).

A nivel global se estima que la prevalencia de hipertiroidismo es de $1-2 \%$ en mujeres y del $0.1-0.2 \%$ en hombres, siendo sus principales causas la enfermedad de Graves y el bocio multinodular tóxico. Por otro lado, el hipotiroidismo clínico tiene una incidencia de $0.3 \%$, mientras que el hipotiroidismo subclínico se presente en $4.3 \%$ de las personas. La incidencia de hipotiroidismo es mayor en mujeres y ancianos. El bocio puede llegar a alcanzar hasta el $80 \%$ de prevalencia en poblaciones con déficit de yodo, principalmente en zonas montañosas del Sureste de Asia, América del Sur, África Central (Rosero López, 2020, pág. 4).

En Ecuador las alteraciones tiroideas son de variada incidencia, la morbilidad de hipotiroidismo e hipertiroidismo se encuentran cercano al $8 \%$ en personas adultas. La 
tasa de prevalencia de hipertiroidismo congénito es de $15 \%$ en recién nacidos. A nivel nacional el cáncer de tiroides se ha incrementado en los últimos años, siendo el segundo más frecuente en mujeres, después del de mama. Entre las patologías tiroideas más frecuentes en las zonas andinas y rurales del Ecuador son el hipotiroidismo y el bocio, su incidencia es aproximadamente es del 5 al $8 \%$ con mayor evidencia en mujeres y mayores de 65 años (Cando-Brito, Rodríguez-Vinueza, Escobar-Arrieta, \& Toaquiza-Aguagallo, 2020, pág. 468).

El diagnóstico y tratamiento adecuado del hipertiroidismo depende del reconocimiento de los signos y síntomas de la enfermedad y la determinación de la etiología. El estudio diagnóstico comienza determinando los niveles de la hormona estimulante de la tiroides (TSH). Cuando los resultados de la prueba son inciertos, la medición de la absorción de radionucleidos ayuda a distinguir entre las posibles causas. Cuando la tiroiditis es la causante, el tratamiento sintomático por lo general es suficiente porque el hipertiroidismo en este caso suele ser transitorio. La enfermedad de Graves, bocio tóxico multinodular y el adenoma tóxico puede tratarse con yodo radiactivo, medicamentos antitiroideos o cirugía. La tiroidectomía es una opción cuando otros tratamientos han fallado o están contraindicados, o cuando un bocio está causando síntomas compresivos (Zúñiga Cruz, 2019, pág. 1).

\section{Función de la Hormona Tiroides}

Las funciones que desempeñan las hormonas tiroideas son bastantes amplias y necesarias para el funcionamiento del organismo, participando así en la regulación del metabolismo de los hidratos de carbono y lípidos, con efecto en el crecimiento, en el sistema cardiovascular, respiratorio, digestivo, sistema nervioso central, sexual e incluso se describen efectos sobre otras glándulas endocrinas (PALMA MERA, 2020, pág. 8).

\section{Importancia de las Glándulas tiroides}

La glándula tiroides se encuentra localizada a nivel del cuello justo por delante de la tráquea. Produce unas hormonas llamadas Tiroxina (T4) y Triyodotiroxina (T3). Estas hormonas actúan a nivel de la mayoría de los tejidos del cuerpo y son necesarias para las etapas de crecimiento y desarrollo del Sistema Nervioso Central. Entre los componentes que forman estas proteínas se encuentra el yodo, elemento importante para su producción. Esta glándula segrega hormonas de gran importancia, que controlan el metabolismo, y por lo tanto, las diversas funciones fisiológicas de nuestro organismo. Además, Interviene en el control, además, de otras glándulas de importancia, como son la hipófisis y el hipotálamo, a lo que se lo denomina eje hipotalámico-. Hipofisario-tiroideo (PALMA MERA, 2020, pág. 8).

\section{Enfermedad de Graves}

La enfermedad de Graves es un trastorno autoinmunitario frecuente que afecta a numerosos órganos y tejidos y cuyo factor esencial es la autoinmunidad contra el receptor de TSH (TSHR). En la mayoría de los pacientes, los anticuerpos contra el TSHR (TR-Ab) circulantes estimulan la tiroides tras su unión al receptor, siendo en definitiva el hipertiroidismo la manifestación más común de la enfermedad de Graves. En poblaciones con ingesta adecuada de yodo, esta enfermedad es la causa dominante de hipertiroidismo, representando alrededor de un $80 \%$ del total de casos de tirotoxicosis. La presentación de los signos de la tiroides, los ojos y la piel se denomina tríada de Graves. En cada caso particular, la enfermedad tiroidea y los fenómenos infiltrantes sobrevienen de forma aislada o conjuntamente, pero siguiendo ciclos en buena medida independientes. El aspecto histológico de la tiroides se diferencia del de la tiroiditis autoinmunitaria por la presencia de un infiltrado linfocítico más diseminado. Sin embargo, en la enfermedad de Graves, el 
hipertiroidismo se desarrolla en presencia de cierto grado de tiroiditis autoinmunitaria y, en última instancia, puede ser reemplazado a largo plazo por hipofunción tiroidea. En cambio, en ocasiones el hipertiroidismo sobreviene en pacientes con tiroiditis de Hashimoto preexistente. Es posible que ambas enfermedades se den en la misma familia, lo que indica su estrecha interrelación (Zúñiga Cruz, 2019, págs. 13-14).

\section{Tabla 1. Patologías de la tiroides}

\begin{tabular}{|c|c|}
\hline Bocio & $\begin{array}{l}\text { El proceso comienza cuando el hipotálamo, una zona en la base del cerebro que actúa } \\
\text { como termostato para todo el sistema, le indica a la hipófisis que fabrique una hormona } \\
\text { conocida como tirotropina. La hipófisis, ubicada también en la base del cerebro, libera una } \\
\text { determinada cantidad de tirotropina, dependiendo de la cantidad de tiroxina y T-3 que } \\
\text { tengas en la sangre. La glándula tiroides, a su vez, regula su producción de hormonas } \\
\text { según la cantidad de tirotropinaque reciba de la hipófisis. Tener bocio no necesariamente } \\
\text { implica que la glándula tiroides no está funcionando normalmente. Incluso cuando está } \\
\text { agrandada, la tiroides puede producir cantidades normales de hormonas. Sin embargo, } \\
\text { podría ser que produzca una cantidad excesiva o muy pequeña de tiroxina y T-3. }\end{array}$ \\
\hline Hipotiroidismo & $\begin{array}{l}\text { Es el trastorno más común de la glándula tiroides. Es una situación clínica caracterizada } \\
\text { por déficit de producción de hormonas tiroideas. Se clasifica en Hipotiroidismo primario: } \\
\text { cuando la alteración se encuentra en la glándula tiroides. Hipotiroidismo secundario: } \\
\text { cuando la alteración ocurre en la glándula hipófisis o en el hipotálamo. Por otro lado, el } \\
\text { hipotiroidismo sub-clínico es una enfermedad asintomática en la mayoría de los casos, en } \\
\text { la que la reducción del funcionamiento de la tiroides no es tanto como para que aparezcan } \\
\text { signos clínicos. De acuerdo a la causa que originó el trastorno, el hipotiroidismo puede ser: } \\
\text { Por deficiencia de yodo. Hipotiroidismo idiopático. En el que se desconoce la causa de la } \\
\text { enfermedad Hipotiroidismo postablativo. Posterior a la extirpación quirúrgica de la } \\
\text { glándula tiroides o después de la terapia con yodo radioactivo. Tiroiditis. Inflamación de la } \\
\text { glándula tiroides producida por fenómenos autoinmunes, que se llama tiroiditis linfocítica } \\
\text { crónica o tiroiditis de Hashimoto. Defectos en el desarrollo de la glándula tiroides. Son } \\
\text { causa común en los primeros meses de vida }\end{array}$ \\
\hline Nódulos Tiroideos & $\begin{array}{l}\text { La mayoría de los nódulos son parcialmente quísticos con un componente sólido, los } \\
\text { nódulos puramente quísticos representan cerca del } 1 \% \text { de todos los nódulos. El cáncer de } \\
\text { tiroides tiene una tasa hombre mujer de } 1 \text { a } 2.5 \text {. El } 74 \% \text { de los nuevos diagnósticos son } \\
\text { mujeres y el cáncer de tiroides es el octavo cáncer más común diagnosticado en mujeres. } \\
\text { La prevalencia incrementa con la edad, el riesgo de malignidad es mucho mayor en } \\
\text { hombres. La exposición a radiación de la cabeza y el cuello en edades tempranas } \\
\text { predispone a que el } 10 \text { al } 40 \% \text { de aquellas personas desarrolle nódulos tiroideos en los } \\
\text { próximos } 5 \text { a } 30 \text { años. La exposición a radiaciones ionizantes causa que los nódulos se } \\
\text { desarrollen a una tasa del } 2 \% \text { anual. }\end{array}$ \\
\hline Cáncer de Tiroides & $\begin{array}{l}\text { El cáncer papilar y el cáncer folicular son el cáncer de tiroides más frecuentes, con una } \\
\text { tasa de supervivencia a los } 10 \text { años del } 98 \% \text { para el cáncer papilar, del } 92 \% \text { para el cáncer } \\
\text { folicular y del } 13 \% \text { para el cáncer anaplasico. El cáncer de tiroides tiene peor pronóstico } \\
\text { en sujetos jóvenes (menor de } 20 \text { años) o en ancianos (mayor de } 65 \text { años), es dos veces más } \\
\text { frecuente en las mujeres que los hombres, pero tiene peor pronóstico en el sexo masculino. }\end{array}$ \\
\hline Hipertiroidismo & $\begin{array}{l}\text { Se conoce como tiroides hiperactiva. Ocurre cuando la glándula tiroides produce y libera } \\
\text { demasiada hormona tiroidea. Afecta su ritmo cardíaco y la función de sus otros órganos. } \\
\text { También afecta sus músculos, huesos y ciclos menstruales (en el caso de las mujeres). En } \\
\text { más del } 70 \% \text { de los casos, el hipertiroidismo es causado por la enfermedad de Graves. } \\
\text { Con la enfermedad de Graves, el sistema inmunitario estimula la tiroides y hace que } \\
\text { produzca demasiada hormona. Los médicos piensan que la enfermedad de Graves puede } \\
\text { ser hereditaria. Es más común entre las mujeres jóvenes. }\end{array}$ \\
\hline Orbitopatía Tiroidea & $\begin{array}{l}\text { La orbitopatia tiroidea }(\mathrm{OT}) \text { es la primera causa de patología orbitaria, con una incidencia } \\
\text { que oscila entre el } 50 \text { y } 60 \% \text { en la consulta de un cirujano de órbita. Es conocida también } \\
\text { con el nombre de oftalmopatia de Graves por su asociación con la enfermedad de Graves, } \\
\text { considerándose la manifestación extratiroidea más frecuente de esta enfermedad, aunque } \\
\text { puede presentarse en pacientes sin historia pasada o presente de hipertiroidismo } \\
\text { (eutiroideos), en pacientes hipotiroideos y en la tiroiditis de Hashimoto. }\end{array}$ \\
\hline
\end{tabular}

Fuente: Elaboración Propia. Tomado de (Romo, y otros, 2019). 
Tabla 2. Principales Efectos Fisiológicos de las Hormonas Tiroideas

\begin{tabular}{|c|l|}
\hline Metabolismo de Lípidos & $\begin{array}{l}\text { Modifican la síntesis, movilización y degradación de los lípidos. Disminuyen la } \\
\text { concentración plasmática de colesterol, ya que aumentan los receptores de } \\
\text { lipoproteínas de baja densidad (LDL) en las células hepáticas, lo que favorece su } \\
\text { depuración del plasma. Aumentan la lipólisis por su efecto a nivel de los } \\
\text { receptores B3 de los lipocitos. }\end{array}$ \\
\hline Metabolismo de Lípidos & Aumento de la lipogénesis por aumento de las enzimas lipogénicas \\
\hline Metabolismo de carbohidratos & $\begin{array}{l}\text { Aumento de la gluconeogénesis hepática al aumentar los sustratos y las enzimas. } \\
\text { Aumento de la absorción intestinal de glucosa. }\end{array}$ \\
\hline Metabolismo de proteínas & $\begin{array}{l}\text { Aumento de la proteólisis, lo que aumenta los aminoácidos para el proceso de } \\
\text { gluconeogénesis hepática. }\end{array}$ \\
\hline Termogénesis & $\begin{array}{l}\text { Aumento de UCP-1 (termogenina) en tejido adiposo pardo, lo que ocasiona que } \\
\text { el gradiente de protones en la membrana mitocondrial interna se disipe en forma } \\
\text { de calor. Aumento de la actividad de la ATPasa Na+ -K+ y ATPasa Ca++ } \\
\text { (SERCA) en el músculo esquelético, lo que genera calor. }\end{array}$ \\
\hline Sistema Cardiovascular & $\begin{array}{l}\text { Efecto cronotrópico positivo: aumento de la sensibilidad de los receptores beta a } \\
\text { las catecolaminas, lo que aumenta la frecuencia cardiaca. Efecto inotrópico } \\
\text { positivo: aumento de la expresión de la cadena pesada de alfa miosina y el } \\
\text { aumento de la sensibilidad de los receptores beta a las catecolaminas, lo que } \\
\text { aumenta la contractilidad cardiaca. }\end{array}$ \\
\hline
\end{tabular}

Fuente: (de Calvo \& de Santos, 2020)

\section{Metodología}

La metodología utilizada para el presente trabajo de investigación, se enfoca hacia una metodología enfocada hacia la necesidad de indagar en forma precisa y coherente una situación. Enmarcada dentro de una revisión bibliográfica de tipo documental, ya que nos vamos a ocupar de temas planteados a nivel teórico como es causas y consecuencias de los trastornos de la tiroides. La técnica para la recolección de datos está constituida por materiales impresos, audiovisuales y electrónicos, estos últimos como Google Académico, PubMed, entre otros. La información aquí obtenida será revisada para su posterior análisis.

\section{Resultados}

\section{Causas y consecuencias de Hipotiroi- dismo}

\section{Causas}

- Espontáneo: tiroiditis crónica autoinmune (Hashimoto) cuya prevalencia aumenta con la edad.
- latrogénico: subtratamiento de hipotiroidismo postratamiento de hipertiroidismo sobre todo con lodo radioactivo o cirugía.

- Drogas: amiodarona, litio y otras medicaciones con iodo.

\section{Consecuencias}

- Niveles de TSH mayor de 10 mUI/L.

- Género femenino.

- Anticuerpos Anti-TPO positivos (Yeza \& Noemí, 2020, pág. 13).

\section{Causas y consecuencias de Hipertiroi- dismo}

Al contrario que en el hipotiroidismo, esta afección está causada por el exceso de producción de hormonas tiroideas. Se calcula que el $15 \%$ de todos los Hipertiroidismos se presentan en mayores de 60 años y, en este caso, afecta a una mayor proporción de hombres que de mujeres. Entre los síntomas habituales del Hipertiroidismo se encuentran la taquicardia, las palpitaciones, el temblor, el exceso de calor, la sudoración, la pérdida de peso repentina, la dificultad para concentrarse o dormir y el nerviosismo

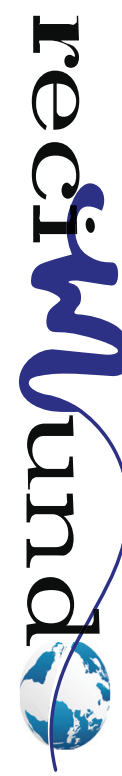


o irritabilidad.

Entre los factores de riesgo del hipertiroidismo se incluyen los siguientes:

- Antecedentes familiares, especialmente de la enfermedad de Graves.

- Sexo femenino.

- Antecedentes médicos personales de determinadas enfermedades crónicas, como diabetes tipo 1, anemia perniciosa e insuficiencia suprarrenal primaria (David \& Cuvi, 2019, pág. 14).

\section{Consecuencias}

- Problemas cardíacos. Algunas de las complicaciones más serias del hipertiroidismo involucran el corazón. Estas incluyen: un ritmo cardíaco rápido, un trastorno del ritmo cardíaco, llamado fibrilación auricular, que aumenta el riesgo de tener un accidente cerebrovascular e insuficiencia cardíaca congestiva, una enfermedad en la cual el corazón no puede hacer circular la cantidad suficiente de sangre para cumplir las necesidades del cuerpo.

- Huesos de cristal. El hipertiroidismo que no se trata también puede llevar a tener huesos frágiles (osteoporosis). La fortaleza de tus huesos depende parcialmente de la cantidad de calcio y otros minerales que estos contengan. Una cantidad excesiva de hormona tiroidea interfiere en la capacidad del cuerpo de incorporar calcio a los huesos.

- Problemas en la vista. Las personas que sufren la oftalmopatía de Graves desarrollan problemas en la vista, incluyendo protrusión, ojos hinchados o rojos, sensibilidad a la luz y visión borrosa o doble. Si no se tratan, estos problemas pueden llevar a la pérdida de la visión (David \& Cuvi, 2019, págs. 14-15).

\section{Nódulo tiroidea \\ Causas}

Los factores predisponentes son: deficiencia de yodo, el efecto de la hormona estimulante de la tiroides, tabaquismo, emba- razo, multiparidad, historia de exposición a radiación ionizante de cabeza y cuello (Rosero López, 2020, pág. 25). Knudsen y colaboradores evaluaron una cohorte de 4.649 daneses y encontraron como más importantes el sexo femenino, la edad avanzada y el tabaquismo; 94\% de los casos ocurrieron en mujeres. Se puede presentar en todas las edades, con mayor incidencia entre la tercera y cuarta décadas de la vida. Otras condiciones asociadas por diferentes autores al nódulo tiroideo son la historia de irradiación a la cabeza y el cuello, la deficiencia de yodo, el embarazo y la multiparidad (Román-González, Restrepo Giraldo, Alzate Monsalve, Vélez, \& Gutiérrez Restrepo, 2013, pág. 198).

\section{Consecuencias}

El pronóstico de los nódulos malignos tras la cirugía y el tratamiento con yodo radiactivo depende del tipo de carcinoma, el tamaño del nódulo, la amplitud de la resección y si hay extensión metastásica. La frecuencia de complicaciones asociadas al procedimiento quirúrgico varía según la experiencia en los diferentes centros; las complicaciones incluyen hipoparatiroidismo transitorio o definitivo, lesión del nervio laríngeo recurrente, necesidad de traqueostomía y lesión de estructuras vasculares cervicales (Román-González, Restrepo Giraldo, Alzate Monsalve, Vélez, \& Gutiérrez Restrepo, 2013, pág. 204).

\section{Cáncer de tiroides}

\section{Causas}

El factor de riesgo más importante es la exposición de la glándula tiroides a la radiación. Menos de 1 de cada 10 nódulos resultan ser cáncer den tiroides (Rosero López, 2020, pág. 26). Los factores de riesgo incluyen historia personal o familiar de cáncer de tiroides, ciertas enfermedades con predilección genética que tienden a desarrollar cáncer de tiroides y previa exposición a irradiación. En la historia la exposición a 
radiación, hasta el 9\% de todos los casos de cáncer de tiroides está relacionado con exposición directa; el punto crítico está en pacientes pediátricos menores de 15 años (Chaparro \& Meza, 2020, pág. 474).

\section{Consecuencias}

El paciente consulta por deformidades del cuello "abultamiento" que corresponde a masas o nódulos, de crecimiento rápido, dolor secundario, sangrado dentro un nódulo o adenopatía cervical, síntomas de disfagia, disfonía o disnea. En general se presentan en enfermedades avanzadas por compromiso de estructuras vecinas.

Aun con función tiroidea normal, el no tratamiento y el crecimiento a través de la cápsula pueden llevar a dificultad respiratoria y crecimiento de cadenas ganglionares laterales. Tanto los cambios de la voz como la ronquera son un claro signo de cáncer de tiroides con invasión del nervio laríngeo (Chaparro \& Meza, 2020, pág. 474).

\section{Conclusiones}

Las hormonas tiroideas son muy importantes, ya que regulan muchas funciones y procesos metabólicos, para que las hormonas tiroideas funciones normalmente, es necesario que la glándula tiroidea se desarrolle normalmente, y para que esto suceda es necesario que haya un soporte nutricional de yodo adecuado, la propia autorregulación tiroidea, el desarrollo normal de los procesos controlados y regulados por el hipotálamo-hipofisario, entre otros. Y esto es importante, ya que el eje hipotálamo-hipófisis-tiroides, regulan las hormonas tiroideas, que permiten mantener un aporte de hormonas adecuado al sistema nervioso, el exceso o deficiencia de estas hormonas pueden producir alteraciones criticas en la vida intrauterina, los primeros años de vida, adolescencia y la etapa adulta.
Aquí hemos podido desarrollar y poder observar que los trastornos de la tiroides pueden producir diferentes patologías, es por ello que su diagnostico es importante, ya que en muchos casos son asintomáticos, pero pueden estar presentes, pudiendo desarrollar o avanzar a patologías mas delicadas como el cáncer, por complicaciones. De allí que el diagnostico oportuno y su tratamiento, son fundamentales para detener el avance de algunas de las patologías asociadas o el problema originario. De igual manera es preciso controlar aquellos factores de riesgo como obesidad, tabaquismo, patrones alimentarios o sedentarios familiares, para controlar un avance de cualquiera de estas patologías, ya que las consecuencias son diversas y pueden llegar a ser muy limitantes.

\section{Bibliografía}

Cando-Brito, V. M., Rodríguez-Vinueza, V., Escobar-Arrieta, S., \& Toaquiza-Aguagallo, N. (2020). Determinación de alteraciones tiroideas en pacientes voluntarios, de un dispensario de salud ocupacional, Riobamba-Ecuador. Polo del Conocimiento, 5(5), 465-483.

Chaparro, J. A., \& Meza, J. (2020). CÁNCER DE TIROIDES. UTP.

David, A., \& Cuvi, C. (2019). Determinación de tsh, t3, t4, antiperoxidasa y antitiroglobulina como ayuda diagnóstica de trastornos tiroideos.

de Calvo, O. L., \& de Santos, L. (2020). EXPERTOS EN FISIOLOGÍA: RESUMEN DE LO QUE DEBES SABER DE LAS HORMONAS TIROIDEAS. Revista Médico Científica, 33(2), 31-45.

Palacio, M., Añez, R., Núñez, T., Mejía, E., Alcibar, R., Rojas, R., \& Maldonado, S. (2017). Disfunción tiroidea subclínica. Medicina Interna, 33(4), 197 207

PALMA MERA, G. C. (2020). Disfunción tiroidea subclínica y variación del peso corporal en pacientes de 25-40 años atendidos en el Centro Medico "Buen Vivir" Cantón Montecristi 2019.

Román-González, A., Restrepo Giraldo, L., Alzate Monsalve, C., Vélez, A., \& Gutiérrez Restrepo, J. (2013). Nódulo tiroideo, enfoque y manejo. Revisión de la literatura. Iatreia, 26(2), 197-206.

Romo, M. A., Montes, P., Burgos, M., Morillo, E.,

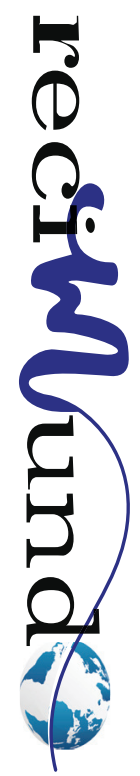


ÑO, J. D.

López, E., \& Mero, M. (2019). Aspectos fisiopatológicos en pacientes con problemas de tiroides. Polo del Conocimiento: Revista científico-profesional, 4(5), 52-68.

Rosero López, J. D. (2020). lesiones tiroideas asociada a la exposición de radiación ionizante en el personal de salud.
Yeza, G., \& Noemí, E. (2020). Prevalencia de disfunción tiroidea y su relación con el perfil lípidico en pacientes ambulatorios que concurren al Hospital Regional de Encarnación.

Zúñiga Cruz, R. (2019). Eficacia del uso de Yodoterapia versus otras opciones terapéuticas en pacientes con hipertiroidismo en el servicio de Medicina Interna del Hospital Alejandro Dávila Bolaños, periodo 1 enero-31 de noviembre del 2018.

\section{CitAR ESTE ARTICULO:}

Zambrano Palacios, F. M., Soledispa Zambrano, M. L., Demera Reyna, G. M., \& Alvarado Cedeño, J. D. (2021). Causas y consecuencias de los trastornos de la tiroides. RECIMUNDO, 5(3), 424-432. https://doi.org/10.26820/recimundo/5. (3).sep.2021.424-432

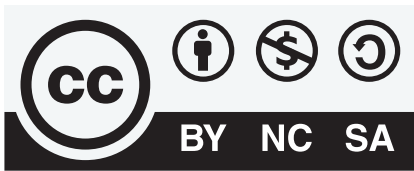

CREATIVE COMMONS RECONOCIMIENTO-NOCOMERCIAL-COMPARTIRIGUAL 4.0 